\title{
CANEUS2006-11035
}

\section{THERMOELASTIC DAMPING IN VIBRATING BEAM ACCELEROMETER: A NEW THERMOELASTIC FINITE ELEMENT APPROACH}

\author{
S. Lepage * \\ University of Liege \\ B-4000 Liege \\ Belgium \\ Email: slepage@ulg.ac.be
}

\author{
O. Le Traon \\ ONERA \\ 92322 Chatillon Cedex \\ France
}

\author{
I. Klapka \\ Open-Engineering \\ B-4031 Angleur \\ Belgium
}

\author{
S. Masson \\ ONERA \\ 92322 Chatillon Cedex \\ France
}

\author{
J.C. Golinval \\ University of Liege \\ B-4000 Liege \\ Belgium
}

\begin{abstract}
In order to respond to the demand of accurate miniature inertial navigation systems, ONERA has been working on the design of a vibrating beam accelerometer called the Vibrating Inertial Accelerometer (VIA). The accuracy of the VIA is directly related to the thermoelastic quality factor of its sensitive element, which is a beam made of quartz. In this work, thermopiezoelectric finite element analyses of the beam are carried out in order to determine the thermoelastic quality factor. These finite element results are compared to the analytical and experimental quality factors. Due to their inherent restrictive assumptions, analytical models overestimate the quality factor while the finite element results are in good agreement with the experimental values. As the finite element model allows to take into account the real geometry of the beam and the piezoelectricity of the material, it allows to quantify more precisely the thermoelastic quality factor.
\end{abstract}

\footnotetext{
*Address all correspondence to this author.
}

\section{INTRODUCTION}

Thermoelastic damping has been identified as an important loss mechanism in numerous high-Q micro-resonators, see for example Refs. [1-5]. The ability to accurately model and predict energy loss due to the thermoelastic effects is therefore a key requirement in order to improve the performance of high-Q resonators. Although most studies of thermoelastic quality factor till date have been based on analytical models, which are subject to very restrictive assumptions so that they are not sufficiently accurate to predict the behavior of complex 3-D structures. In this paper, a finite element formulation has been developed in order to analyze the behavior of systems that are not analytically tractable.

The resonator devices used in this study are accelerometers fabricated at ONERA. In order to respond to the demand of accurate miniature inertial navigation systems, ONERA has been working on the design of a vibrating beam accelerometer called the Vibrating Inertial Accelerometer (VIA) [6]. The present applications of this device are the guidance and the attitude control of tactical missiles as well as aircraft inertial navigation. The ac- 


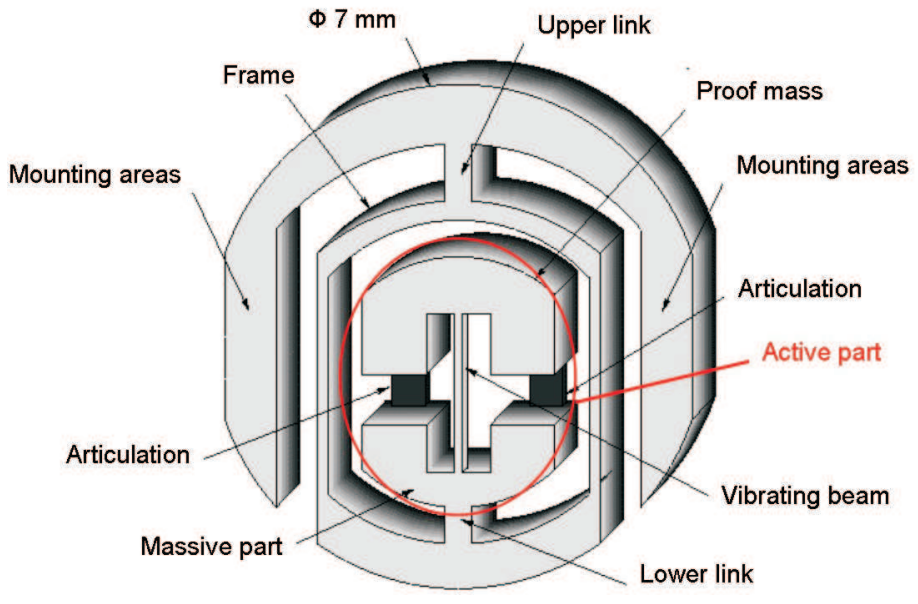

Figure 1. VIA DESIGN WITH THE DECOUPLING FRAME

curacy of the VIA is directly related to the quality factor of its sensitive element, which is a beam made of quartz. The aim of this paper is to study the influence of the thermoelastic effects on the behavior of the VIA.

Firstly, the VIA is introduced and the importance of the thermoelastic effects on its performances is highlighted. Then, thermoelastic damping in beam resonators is briefly reviewed and the thermo-piezoelectric finite element formulation is derived. Finally, finite element analyses are carried out and the results are compared to the analytical and experimental quality factors.

\section{THE VIBRATING INERTIAL ACCELEROMETER}

The Vibrating Inertial Accelerometer (VIA) [6-8] is a Vibrating Beam Accelerometer (VBA) made of monocrystalline quartz. Its concept is based on the resonance frequency shift of a beam when submitted to axial stresses induced by acceleration. More precisely, in the VIA design, a micrometric beam (cross section $30 \mu m \times 60 \mu m$, length $2.26 \mathrm{~mm}$ ) is clamped at one of its ends and is connected to proof mass at the other (see Fig. 1). When an acceleration is applied along the sensitive axis of the sensor (perpendicular to the transducer plane), the proof mass generates an axial stress into the beam, which modifies its bending resonance frequency.

As quartz is a piezoelectric material, it is possible to actuate and detect the oscillations of the beam by metallic electrodes which are deposited on it. An electronic oscillator, with gain and phase control, is used to excite the beam at its resonance. The output of VIA is thus the frequency of the oscillator signal, and its variations represent the applied acceleration. Bias stability, i.e. beam frequency without acceleration, requires a resonator with high quality factor, in order to reduce the sensitivity of electronic phase drift. The quality factor $(Q)$ is defined by the ratio of the stored energy in the resonator $(W)$ and the total dissipated energy per cycle of vibration $(\Delta W)$ :

$$
Q=2 \pi \frac{W}{\Delta W}
$$

Q is affected by several loss mechanisms, which are extrinsic (losses by gas damping, losses into support,...) or intrinsic (viscosity, thermoelastic damping,...). The global quality factor $Q_{\text {total }}$ can be express as [9] :

$$
Q_{\text {total }}^{-1}=\sum_{i} Q_{i}^{-1}=Q_{\text {surface }}^{-1}+Q_{\text {support }}^{-1}+Q_{\text {visco }}^{-1}+Q_{T E D}^{-1}
$$

where $Q_{\text {surface }}$ represents the surface losses (such as gas damping), $Q_{\text {support }}$ are the energy losses due to the fixation to the support, $Q_{\text {visco }}$ corresponds to losses due to intrinsic losses and $Q_{T E D}$ is the effect of thermoelastic damping

In the VIA, extrinsic losses have been decreased as much as possible. Indeed, gas damping is avoided by operating under vacuum ( $\mathrm{p}=0.1 \mathrm{mbar}$ ). Moreover, a specific insulation frame has been developed in order to reduce the losses out of the quartz structure. Finite element analyses show that, due to this frame, less than $10^{-8}$ of the whole energy is dissipated into the support, and really high quality factors are allowed. Lastly, due to the quality of quartz crystal, viscosity losses can be neglected.

Thus, intrinsic losses, and especially thermoelastic damping are now the main contribution to energy losses and limit the VIA quality factor: $Q_{t o t a l}=Q_{T E D}$. Experimental quality factor $Q_{\text {total }}$ is about 13000 , whereas Zener's thermoelastic model [10] evaluates $Q_{T E D}$ around 17000 . This difference can be explained by the strong assumptions of the analytical model (such as rectangular beam, isotropic solid,...) which are not satisfied in the case of the VIA beam. So, a finite element approach has been developed in order to have a better understanding of the thermoelastic damping, and to improve the design of future accelerometers.

\section{THERMOELASTIC DAMPING}

The basic notions of thermoelasticity are well known [11]. In isotropic solids with a positive thermal expansion coefficient, an increase of temperature creates an expansion and inversely, a decrease of temperature produces a compression. Similarly, an expansion lowers the temperature and a compression raises the temperature. Therefore, when a thermoelastic solid is set in motion, it is taken out of equilibrium, having an excess of kinetic and potential energy. The coupling between the strain and the temperature fields induces an energy dissipation mechanism which causes the system to return to its static equilibrium. The 
relaxation of the thermoelastic solid is achieved through the irreversible flow of heat driven by local temperature gradients that are generated by the strain field. Thermoelastic damping results from this dissipation which is not always measurable. When the vibration frequency is much lower than the relaxation rate, the solid is always in thermal equilibrium and the vibrations are isothermal. On the other hand, when the vibration frequency is much higher than the relaxation rate, the system has no time to relax and the vibrations are adiabatic. Hence, it is only when the vibration frequency is of the order of the relaxation rate that the energy loss becomes appreciable.

\section{Analytical models}

Zener [10] was the first to develop expressions to approximate the thermoelastic damping. His theory is based on an extension of Hooke's law involving stress $\sigma$, strain $\varepsilon$ as well as their first time derivatives $\dot{\sigma}$ and $\dot{\varepsilon}[10]$ :

$$
\sigma+\tau_{\varepsilon} \dot{\sigma}=E_{R}\left(\varepsilon+\tau_{\sigma} \dot{\varepsilon}\right)
$$

This model is called the "Standard Anelastic Solid" model. The three parameters $\tau_{\varepsilon}, \tau_{\sigma}$ and $E_{R}$ have the following physical interpretation:

- $\tau_{\varepsilon}$ is the relaxation time at which the stress relaxes exponentially when the strain is kept constant.

- $\tau_{\sigma}$ is the relaxation time at which the strain relaxes exponentially when the stress is kept constant.

- $E_{R}$ is the elastic modulus after all relaxations have occurred.

The unrelaxed value of the elastic modulus $E_{U}$ can be defined using the three previous parameters:

$$
E_{U}=E_{R} \frac{\tau_{\sigma}}{\tau_{\varepsilon}}
$$

In order to analyze the characteristics of the solid vibrations, the stress and the strain are considered to vary harmonically at the natural pulsation $\omega_{n}$. The dissipation in the solid can be measured by $Q^{-1}$, the inverse of the quality factor of the resonating structure, which is defined as the fraction of energy lost per cycle:

$$
Q^{-1}=\Delta_{E} \frac{\omega_{n} \tau}{1+\left(\omega_{n} \tau\right)^{2}}
$$

where $\tau=\sqrt{\tau_{\sigma} \tau_{\varepsilon}}$ is the effective relaxation time and $\Delta_{E}=\sqrt{\frac{\tau_{\sigma}}{\tau_{\varepsilon}}}-\sqrt{\frac{\tau_{\varepsilon}}{\tau_{\sigma}}}=\frac{E_{U}-E_{R}}{\sqrt{E_{R} E_{U}}}$ is the relaxation strength.
Thus, the dissipation exhibits a Lorentzian behavior as a function of $\omega_{n} \tau$ with a maximum value of $\Delta_{E} / 2$ when $\omega_{n} \tau=1$. This agrees with the previous qualitative explanation. When the frequency is small compared to the relaxation rate, i.e. $\omega_{n} \tau<<1$, the thermoelastic dissipation is negligible and the oscillations are isothermal. On the other hand, when the frequency is large compared to the relaxation rate, i.e. $\omega_{n} \tau>>1$, the oscillations are adiabatic. Therefore, it is only when the frequency is of the order of the relaxation rate, i.e. $\omega_{n} \tau \approx 1$, that the thermoelastic dissipation takes importance.

For a beam in flexion, assuming that the relaxation occurs only through the first transverse conduction mode and that the thermoelastic natural frequency $\omega_{n}$ can be approximated by the isothermal frequency $\omega_{o, n}$, the inverse of the quality factor for a thermoelastic flexural beam resonator can be expressed as follows

$$
Q^{-1}=\frac{E \alpha^{2} T_{o}}{C_{v}} \frac{2 \zeta^{2} / \pi^{2}}{1+\left(2 \zeta^{2} / \pi^{2}\right)^{2}}
$$

where $E$ is the Young modulus, $\alpha$ is the heat expansion coefficient, $C_{v}$ is the heat capacity at constant volume, $T_{o}$ is the reference temperature and $\zeta$ is a dimensionless parameter which depends on the thermal diffusivity $\chi=\kappa / C_{v}$ where $\kappa$ is the thermal conductivity, the beam thickness $b$ and the isothermal frequency $\omega_{o, n}: \zeta=b \sqrt{\frac{\omega_{o, n}}{2 \chi}}$.

Lifshitz and Roukes (LR) [12] proposed an analysis based on the same fundamental physics but in which the transverse temperature profile is more accurately modeled. Their model gives the following expression for the inverse of the quality factor:

$$
Q^{-1}=\frac{E \alpha^{2} T_{o}}{C_{v}}\left(\frac{6}{\zeta^{2}}-\frac{6}{\zeta^{3}} \frac{\sinh \zeta+\sin \zeta}{\cosh \zeta+\cos \zeta}\right)
$$

The quality factors predicted by LR model differ from Zener's ones by between $2 \%$ and $20 \%$ depending on the value of the dimensionless parameter $\zeta$. Indeed, it can be showed that the quality factor given by equation (7) is bounded between two Lorentzians:

$$
\Delta_{E} \frac{2 \sqrt{6}}{5} \mathcal{L}\left(\frac{\zeta^{2}}{\sqrt{24}}\right) \leq Q^{-1} \leq \Delta_{E} \frac{\sqrt{6}}{2} \mathcal{L}\left(\frac{\zeta^{2}}{\sqrt{24}}\right)
$$

where the Lorentzian $\mathcal{L}$ is defined as:

$$
\mathcal{L}(\eta)=\frac{\eta}{1+\eta^{2}}
$$

For small values of $\zeta$, the quality factor tends to its lower Lorentzian bound. While for large values of $\zeta$, it tends to its 
upper Lorentzian bound. Zener's solution corresponds to the following Lorentzian:

$$
Q^{-1}=\mathcal{L}\left(\frac{2 \zeta^{2}}{\pi^{2}}\right)
$$

It results from this comparison that expressions (6) and (7) differ by less than $2 \%$ on the isothermal side of the peak (low $\zeta$ ). While on the adiabatic side of the peak (high $\zeta$ ), the difference can reach $20 \%$. Hence, when considering configurations located on the adiabatic side of the peak, it is better to use LR model than Zener's approximation.

\section{Finite Element Formulation}

The analytical models are based on very restrictive assumptions and can only be used for simple beam-like configurations. Even if some work have been carried out to extend the analytical models to polycrystalline beams [13], laminated beams [14] or uniform rings of rectangular cross-section [15], in order to investigate complex structures (i.e. non rectangular geometry, anisotropic material,...), a numerical approach is required. The finite element method can be used to solve the dynamics of thermo-piezoelectric structures [16].

The thermo-piezoelectric finite element formulation can be derived from Hamilton's variational principle in which mechanical, thermal and electric degrees of freedom are considered simultaneously. The displacement field $\mathbf{u}$, the electric potential $\Phi$ and the temperature increment $\theta$ are related to the corresponding node values $\mathbf{u}_{\mathbf{u}}, \mathbf{u}_{\Phi}$ and $\mathbf{u}_{\theta}$ by the mean of the shape function matrices $\mathbf{N}_{\mathbf{u}}, \mathbf{N}_{\Phi}$ and $\mathbf{N}_{\theta}$

$$
\begin{aligned}
\mathbf{u} & =\mathbf{N}_{\mathbf{u}} \mathbf{u}_{\mathbf{u}} \\
\Phi & =\mathbf{N}_{\Phi} \mathbf{u}_{\Phi} \\
\boldsymbol{\theta} & =\mathbf{N}_{\theta} \mathbf{u}_{\theta}
\end{aligned}
$$

Therefore, the strain field $\varepsilon$, the electric field $\mathbf{E}$ and the thermal field $\mathbf{e}$ are related to this nodal values by the shape function derivative matrices $\mathbf{B}_{\mathbf{u}}, \mathbf{B}_{\Phi}$ and $\mathbf{B}_{\theta}$

$$
\begin{aligned}
\varepsilon & =\mathcal{D} \mathbf{N}_{\mathbf{u}} \mathbf{u}_{\mathbf{u}}=\mathbf{B}_{\mathbf{u}} \mathbf{u}_{\mathbf{u}} \\
\mathbf{E} & =-\nabla \mathbf{N}_{\Phi} \mathbf{u}_{\Phi}=\mathbf{B}_{\Phi} \mathbf{u}_{\Phi} \\
\mathbf{e} & =-\nabla \mathbf{N}_{\theta} \mathbf{u}_{\theta}=\mathbf{B}_{\theta} \mathbf{u}_{\theta}
\end{aligned}
$$

where $\nabla$ is the gradient operator and $\mathcal{D}$ is the derivation operator defined so that $\varepsilon=\mathcal{D} \mathbf{u}$ according to the displacement compatibility equation. The finite element discretisation leads to the fol- lowing dynamic equilibrium equation that governs the thermopiezoelectric behavior of the system :

$$
\begin{gathered}
\left(\begin{array}{ccc}
\mathbf{M}_{\mathbf{u u}} & 0 & 0 \\
0 & 0 & 0 \\
0 & 0 & 0
\end{array}\right) \\
\left(\begin{array}{c}
\ddot{\mathbf{u}}_{\mathbf{u}} \\
\ddot{\mathbf{u}}_{\Phi} \\
\ddot{\mathbf{u}}_{\theta}
\end{array}\right)+\left(\begin{array}{ccc}
0 & 0 & 0 \\
0 & 0 & 0 \\
\mathbf{C}_{\theta \mathbf{u}} & \mathbf{C}_{\theta \Phi} & \mathbf{C}_{\theta \theta}
\end{array}\right)\left(\begin{array}{c}
\dot{\mathbf{u}}_{\mathbf{u}} \\
\dot{\mathbf{u}}_{\Phi} \\
\dot{\mathbf{u}}_{\theta}
\end{array}\right)+ \\
+\left(\begin{array}{ccc}
\mathbf{K}_{\mathbf{u u}} & \mathbf{K}_{u \Phi} & \mathbf{K}_{\mathbf{u} \theta} \\
\mathbf{K}_{\Phi u} & \mathbf{K}_{\Phi \Phi} & \mathbf{K}_{\Phi \theta} \\
0 & 0 & \mathbf{K}_{\theta \theta}
\end{array}\right)\left(\begin{array}{c}
\mathbf{u}_{\mathbf{u}} \\
\mathbf{u}_{\Phi} \\
\mathbf{u}_{\theta}
\end{array}\right)=\left(\begin{array}{c}
\mathbf{F}_{\mathbf{u}} \\
\mathbf{F}_{\Phi} \\
\mathbf{F}_{\theta}
\end{array}\right)
\end{gathered}
$$

where $\mathbf{M}_{\mathbf{u u}}$ is the mass matrix, $\mathbf{C}_{\theta \mathbf{u}}$ and $\mathbf{C}_{\theta \Phi}$ are the damping matrices due to thermo-mechanical and thermo-electric coupling effect, respectively and $\mathbf{C}_{\theta \theta}$ is the damping matrix due to the thermal field. Matrices $\mathbf{K}_{u \Phi}$ and $\mathbf{K}_{\Phi u}$ are the stiffness matrices due to piezoelectric coupling effect. Matrices $\mathbf{K}_{\mathbf{u} \theta}$ and $\mathbf{K}_{\Phi \theta}$ are the stiffness matrices due to thermo-mechanical and thermo-electric coupling, respectively. Matrices $\mathbf{K}_{\mathbf{u u}}, \mathbf{K}_{\Phi \Phi}$ and $\mathbf{K}_{\theta \theta}$ are the stiffness matrices due to mechanical, electric and thermal fields, respectively. Vectors $\mathbf{F}_{\mathbf{u}}, \mathbf{F}_{\Phi}$ and $\mathbf{F}_{\theta}$ are the force vectors due to mechanical, electric and thermal fields, respectively.

Thermoelastic effects modify the quality factor of the response, inducing both damping and resonance frequency shift. In order to quantify the quality factor of a structure, a modal analysis has to be carried out. Equation (17) takes the general form

$$
\mathbf{M} \ddot{\mathbf{q}}+\mathbf{C} \dot{\mathbf{q}}+\mathbf{K q}=0
$$

where $\mathbf{C}$ and $\mathbf{K}$ are non-symmetric matrices. This problem may be transformed into a linear problem of twice the size through a linearization procedure. Partitioning the eigenvectors into electric, thermal and mechanical degrees of freedom and substituting the time derivative of the thermal degrees of freedom by their values, the eigenvalue problem to solve may be rewritten in the form

$$
\begin{array}{r}
\left(\begin{array}{cccc}
-\mathbf{K}_{u u} & -\mathbf{K}_{u \Phi} & -\mathbf{K}_{u \theta} & 0 \\
-\mathbf{K}_{\Phi u} & -\mathbf{K}_{\Phi \Phi} & -\mathbf{K}_{u \Phi} & 0 \\
0 & 0 & -\mathbf{K}_{\theta \theta} & 0 \\
0 & 0 & 0 & \mathbf{M}_{u u}
\end{array}\right) \\
=\lambda\left(\begin{array}{c}
\mathbf{x}_{u} \\
\mathbf{x}_{\Phi} \\
\mathbf{x}_{\theta} \\
\dot{\mathbf{x}}_{u}
\end{array}\right) \\
\left(\begin{array}{cccc}
0 & 0 & 0 & \mathbf{M}_{u u} \\
0 & 0 & 0 & 0 \\
\mathbf{C}_{\theta u} & \mathbf{C}_{\theta \Phi} & \mathbf{C}_{\theta \theta} & 0 \\
\mathbf{M}_{u u} & 0 & 0 & 0
\end{array}\right)\left(\begin{array}{c}
\mathbf{x}_{u} \\
\mathbf{x}_{\Phi} \\
\mathbf{x}_{\theta} \\
\dot{\mathbf{x}}_{u}
\end{array}\right)
\end{array}
$$

If the number of electric, mechanical and thermal degrees of freedom is denoted $n_{\Phi}, n_{u}$ and $n_{\theta}$, respectively, the eigenvalue problem (Eq. 19) has $2 n_{u}$ conjugate complex eigenvalues, $n_{\theta}$ real eigenvalues and $n_{\Phi}$ infinite eigenvalues. The $2 n_{u}$ eigenvalues correspond to the mechanical eigenfrequencies, the $n_{\theta}$ ones 
to the thermal eigenfrequencies and the $n_{\Phi}$ ones to the electric eigenfrequencies.

The quality factor of the nth mode is given by

$$
Q=\frac{\omega_{i}}{2 \omega_{r}}
$$

where $\omega_{r}$ and $\omega_{i}$ are the real and imaginary parts of the nth conjugate complex eigenvalue of Eq. (19). Note that another way to determine the quality factor is to carry out a harmonic analysis and to derive the value of the quality factor from the frequency response function of the structure.

All these finite element developments are implemented in a software called Oofelie ("Object Oriented Finite Element Led by Interactive Executer"). This software is written in $\mathrm{C}++$ language so that it allows to solve multiphysic problems with strong coupling $[17,18]$.

\section{THERMOELASTIC QUALITY FACTOR OF THE VIBRAT- ING INERTIAL ACCELEROMETER}

In order to quantify the performance of the VIA, the thermoelastic quality factor of its sensitive part, a clamped-clamped beam, has to be determine. The beam is made of quartz, which is anisotropic and moreover piezoelectric. Figure 2 shows the geometry of the beam. It is to be noted that the scale in the direction of the beam length is ten times smaller than the one in the other two directions. The cross-section of the beam is a right trapezoid. Due to chemical anisotropic etching of quartz during the manufacturing of transducers, some crystalline planes appear and modify the beam geometry, so that the real cross-section of VIA beams is trapezoidal. The beam vibrates along the direction of the largest sides of the trapezoid. The dimensions are given in Tab. 1. Figure 2 shows also the configuration of the activation electrodes. These electrodes are made of gold and their thickness is of $200 \mathrm{~nm}$.

Table 1. DIMENSIONS OF VIA VIBRATING BEAM

\begin{tabular}{cc}
\hline Data & Dimension $[\mu m]$ \\
\hline Height & 33 \\
Large base & 59 \\
Small base & 54 \\
Length & 2260 \\
\hline
\end{tabular}

Experiments have shown that the quality factor of the VIA is around 13000 at a resonance frequency of about $62 \mathrm{kHz}$. How-

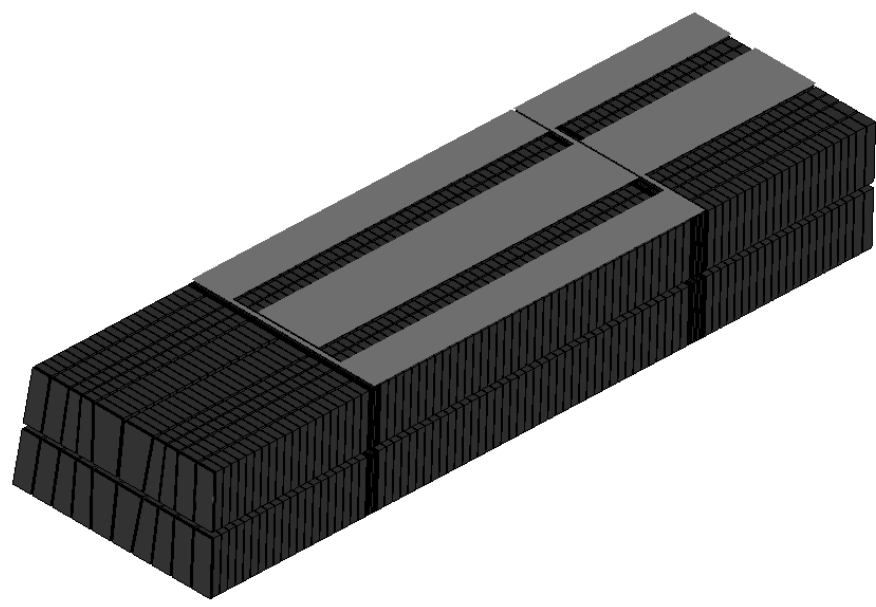

Figure 2. VIA BEAM GEOMETRY WITH ELECTRODE POSITION

ever, using equivalent isotropic material data and a resonance frequency of $62 \mathrm{kHz}$, Zener's model (Eq. 6) gives a quality factor of 16576 , which is nearly $30 \%$ higher than the experimental value. LR model (Eq. 7) gives 14763, which is $12 \%$ different from Zener's quality factor. The difference between the two analytical models has been introduced above. It has been showed that this difference is large when the dimensionless frequency is high. In order to check the influence of the dimensionless frequency, the cantilever configuration, for which the beam is clamped at one end and free at the other one, is considered. In terms of parameters that are used in the analytical models, the cantilever configuration differs from the clamped-clamped one only in its frequency, which is lower. For the cantilever configuration, Zener's quality factor is 2712 while LR model gives 2620. As expected, in this case, the difference between the two analytical models is smaller.

Mathematically, the difference is explained by the inherent approximations assumed in the transverse temperature profile. As in the finite element method, no approximation is made about the transverse temperature profile, the FE results can be considered as the exact solutions. Finite element analyses of both configurations are carried out using exactly the same geometry and material data as those used in the analytical models. FEM quality factors differ from a few percents from LR results. Figure 3 represents the transverse temperature profile assumed in the two analytical models as well as the finite element profile. For both configurations (cantilever and clamped-clamped), LR curve gives a better approximation than Zener's curve. As expected, the two FE curves are different. Indeed, the deformations induced by the bending mode of a cantilever and a clamped-clamped beam are different and due to the thermo-mechanical coupling, the induced temperature distributions are also different. 


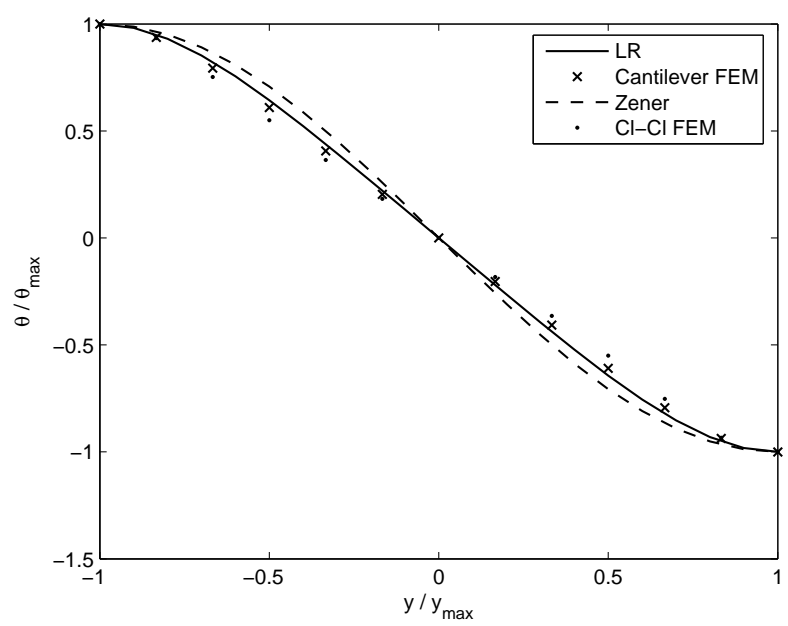

Figure 3. TRANSVERSE TEMPERATURE PROFILE

Even if LR model gives a better approximation than Zener's one, LR quality factor is still more than $10 \%$ higher than the experimental value. This overestimation can be due to the inability of LR model to take into account the real geometry and material behavior. Indeed, the cross-section is trapezoidal, not rectangular, the material is anisotropic, not isotropic. Moreover, piezoelectricity as well as the electrodes can also influence the quality factor. In order to study the effects of all these factors, different finite element analyses are carried out.

Table 2. RECTANGULAR CROSS-SECTION CONFIGURATION RESONANCE FREQUENCY

\begin{tabular}{cll}
\hline Configuration & FEM Fr. [Hz] & Ana. Fr. [Hz] \\
\hline Iso cantilever & 9751 & 9682 \\
Ani cantilever & 9724 & 9682 \\
Iso clamped-clamped & 61871 & 61949 \\
Ani clamped-clamped & 61607 & 61949 \\
\hline
\end{tabular}

Firstly, the effect of anisotropy is taking into account. Tables 2 and 3 summarize the results for the clamped-clamped and cantilever configurations. They show that anisotropy slightly increases the quality factor while it lowers the resonance frequency. However, the anisotropy effect can not explain the difference with the experimental results.

Trapezoidal cross-section finite element models are inves-
Table 3. RECTANGULAR CROSS-SECTION CONFIGURATION QUALITY FACTOR

\begin{tabular}{clll}
\hline Configuration & FEM Q & LR Q & Z Q \\
\hline Iso cantilever & 2640 & 2620 & 2712 \\
Ani cantilever & 2649 & 2620 & 2712 \\
Iso clamped-clamped & 14111 & 14763 & 16576 \\
Ani clamped-clamped & 14157 & 14763 & 16576 \\
\hline
\end{tabular}

tigated. Table 4 lists the results for trapezoidal cross-section configurations. As before, anisotropy decreases the resonance frequency while it increases the quality factor. However, as for rectangular cross-section configurations, quality factors are still $10 \%$ larger than the experimental results.

Table 4. TRAPEZOIDAL CROSS-SECTION CONFIGURATION RESULTS

\begin{tabular}{cllll}
\hline Configuration & FEM Fr. [Hz] & FEM Q & LR Q & Z Q \\
\hline Isotropic & 61992 & 14236 & 14763 & 16576 \\
Anisotropic & 61626 & 14644 & 14763 & 16576 \\
\hline
\end{tabular}

Table 5. VIA STRUCTURE RESULTS

\begin{tabular}{cll}
\hline Configuration & FEM Fr. [Hz] & FEM Q \\
\hline TE w/o elec & 61830 & 15125 \\
TP w/o elec & 62117 & 14363 \\
TE w/ elec & 60815 & 13700 \\
TP w/ elec & 61111 & 13090 \\
\hline
\end{tabular}

Two effects are still to be investigated: piezoelectricity and the influence of the electrodes. Piezoelectricity is known to increase the resonance frequency, what could also affect the quality factor. The electrodes made of gold, which is a really good thermal conductor compared to quartz, will perturb the thermal field and hence, modify the quality factor. Table 
5 gives the quality factor of the VIA for four different models: thermoelastic analysis without electrodes, thermo-piezoelectric analysis without electrodes, thermoelastic analysis with electrodes and thermo-piezoelectric analysis with electrodes. It can be seen that the electrodes decrease the quality factor but also the resonance frequency. Figures 4 and 5 show the temperature increment magnitude distributions corresponding to the bending mode of the VIA beam with and without electrodes, respectively. The electrodes modify the temperature distribution on the surface of the beam creating temperature gradient through the height of the beam so that the quality factor decreases significantly. Piezoelectricity increases the resonance frequency as well as it also slightly lowers the quality factor.

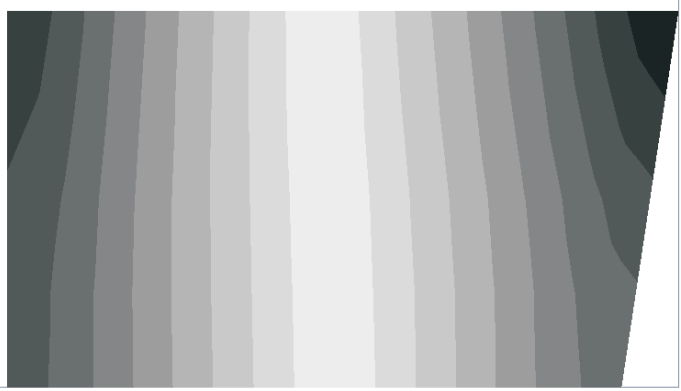

Figure 4. TEMPERATURE INCREMENT MAGNITUDE DISTRIBUTION OF THE VIA BEAM BENDING MODE WITHOUT ELECTRODES

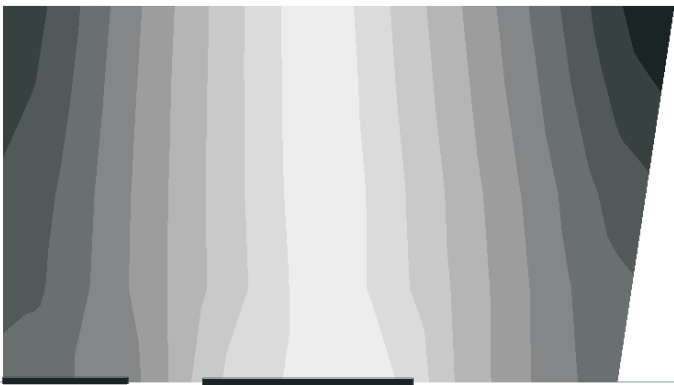

Figure 5. TEMPERATURE INCREMENT MAGNITUDE DISTRIBUTION OF THE VIA BEAM BENDING MODE WITH ELECTRODES
Therefore, when taking into account a model actuated by piezoelectricity, the quality factor as well as the resonance frequency correspond to the experimental values. These analyses show that the electrodes play an important role in the quality factor and that piezoelectricity has to be taken into account in order to accurately determine the resonance frequency.

\section{CONCLUSIONS}

Finite element analyses have been carried out to determine the thermoelastic quality factor of the VIA. It has been showed that analytical models are not sufficient in order to study real complex 3-D structures due to their inherent restrictive assumptions.

In the case of the VIA, finite element analyses have shown the importance of the influence of the gold electrodes on the quality factor and the effect of piezoelectric actuation on the resonance frequency. So that a thermo-piezoelectric finite element model taking into account the gold electrodes gives the same results than the experiment in terms of the quality factor and the resonance frequency.

Thanks to the finite element approach, the different factors that influence the behavior of the VIA have been identified. Now, having a better understanding of the physics involved, the design of the VIA could be modified in order to increase the quality factor and hence, its performance.

\section{ACKNOWLEDGMENT}

The author S. Lepage is supported by the Belgian National Fund for Scientific Research (FNRS), which is gratefully acknowledged. This work is also supported by the Communauté Française de Belgique - Direction Générale de la Recherche Scientifique in the framework Actions de Recherche Concertées (convention ARC 03/08-298).

\section{REFERENCES}

[1] Duwel, A., Gorman, J., Weinstein, M., Borenstein, J., and Ward, P., 2002. "Quality factors of mems gyros and the role of thermoelastic damping". In Proceedings of the 15th International Conference on Microelectromechanical Systems (MEMS), pp. 214-219.

[2] Duwel, A., Gorman, J., Weinstein, M., Borenstein, J., and Ward, P., 2003. "Experimental study of thermoelastic damping in mems gyros". Sensors and Actuators A(103), pp. 70-75.

[3] Houston, B., Photiadis, D., Marcus, M., Bucaro, J., Liu, X., and Vignola, J., 2002. "Thermoelastic loss in microscale oscillators". Applied physics letter, 80(7), February, pp. $1300-1302$. 
[4] Houston, B., Photiadis, D., Vignola, J., Marcus, M., Liu, X., Czaplewski, D., Sekaric, L., Butler, J., Pehrsson, P., and Bucaro, J., 2004. "Loss due to transverse thermoelastic currents in microscale resonators". Materials Science and Engineering A(370), pp. 407-411.

[5] Abdolvand, R., Ho, G., Erbil, A., and Ayazi, F., 2003. "Thermoelastic damping in trench-refilled polysilicon resonators". In Transducers'03, the 12th International Conference on Solid State Sensors, Actuators and Microsystems, pp. 324-327.

[6] Le Traon, O., Janiaud, D., Muller, S., and Bouniol, P., 1998. "The via vibrating beam accelerometer: concept and performance”. In PLANS'98, Position Location and Navigation Symposium.

[7] Le Traon, O., Janiaud, D., and Muller, S. "Monolithic accelerometer transducer". US Patent n 5,962,786 published: 10/05/1999.

[8] Masson, S., Janiaud, D., Le Traon, O., and Muller, S., 2006. "Design and performances of two quartz monolithic vibrating inertial microsensors". In Caneus 2006.

[9] Le Foulgoc, B., Bourouina, T., Le Traon, O., Bosseboeuf, A., Marty, F., Breluzeau, C., Grandchamp, J.-P., and Masson, S., 2006. "Highly decoupled single-crystal silicon resonators: an approach for the intrinsic quality factor". $J$. Micromech. Microeng., 16.

[10] Zener, C., 1937. "Internal friction in solids". Physical Review, 52, August, pp. 230-235.

[11] Nowacki, W., 1986. Thermoelasticity. Pergamon Press, Oxford.

[12] Lifshitz, R., and Roukes, M., 2000. "Thermoelastic damping in micro-and nano-mechanical systems". Physical Review B, 61(8), February, pp. 5600-5609.

[13] Srikar, V., and Senturia, S., 2002. "Thermoelastic damping in fine-grained polysilicon flexural beam resonators". Journal of microelectromechanical systems, 11(5), pp. 499-504.

[14] Bishop, J., and Kinra, V., 1993. "Thermoelastic damping of a laminated beam in flexure and extension". Journal of Reinforced Plastics and Composites, 12, February, pp. 210226.

[15] Wong, S., Fox, C., and McWilliam, S. "Thermoelastic damping of the in-plane vibration of thin silicon rings". Journal of Sound and Vibration, 293(1).

[16] Lepage, S., and Golinval, J.-C., 2005. "Finite element modeling of the thermoelastic damping in microelectromechanical systems". In Acomen 2005, Third International Conference on Advanced Computational Methods in Engineering.

[17] Klapka, I., Cardona, A., and Géradin, M., 1998. “An objectoriented implementation of the finite element method for coupled problems". Revue européenne des Eléments Finis, August.

[18] Klapka, I., Cardona, A., and Géradin, M., 2000. "Inter- preter OOFELIE for PDEs". In European Congress on Computational Methods in Applied Sciences and Engineering, ECCOMAS 2000. 